\title{
On first-order phase transition in microcanonical and canonical non-extensive systems
}

\author{
I. Ispolatov ${ }^{\dagger}$ and E. G. D. Cohen \\ Center for Studies in Physics and Biology, Rockefeller University, 1230 York Ave, New York, NY 10021, USA.
}

\begin{abstract}
Two examples of Microcanonical Potts models, 2-dimensional nearest neighbor and mean field, are considered via exact enumeration of states and analytical asymptotic methods. In the interval of energies corresponding to a first order phase transition, both of these models exhibit a convex dip in the entropy vs energy plot and a region with negative specific heat within the dip. It is observed that in the nearest neighbor model the dip flattens and disappears as the lattice size grows, while in the mean field model the dip persists even in the limit of an infinite system. If formal transitions from microcanonical to canonical ensembles and back are performed for an infinite but non-extensive system , the convex dip in the microcanonical entropy plot disappears.
\end{abstract}

PACS numbers: 05.70.Fh 64.10.+h 64.60.-i 82.60.Qr

\section{INTRODUCTION}

Phase transitions in microcanonical ensembles (ME), particularly in finite systems or long-range interacting systems, which are both nonextensive, have recently become a major subject of theoretical and computational study [1] 8]. Although a lot has been done, in this work we bring up some points which may not be easy to deduce from the literature.

A distinct sign of a ME first-order phase transition is a convex "dip" [2] or "intruder" [7] in an otherwise concave entropy plot (Fig. 1).

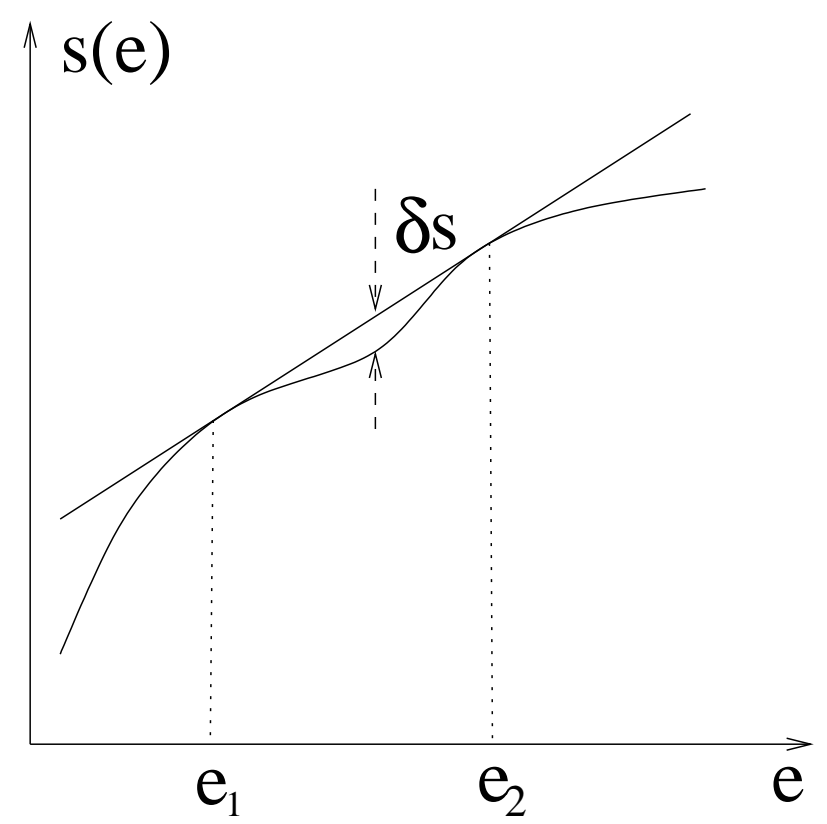

Fig. 1. Sketch of typical microcanonical specific entropy $s(e)$ as a function of the energy per particle $e$ for a small system with a first-order phase transition A convex dip of the depth $\delta s$ can be observed between $e_{1}$ and $e_{2}$.

In the simple case of a system with a one-dimensional thermodynamic space, which is considered in this paper, a convex dip always includes an interval between two points of inflection [3] where a second derivative of the specific entropy is positive and the heat capacity is negative. The convex dip in the entropy plot corresponds to the states of a two-phase coexistence; these states are unstable in the canonical ensemble $(\mathrm{CE})$ and can not be accessed by equilibrium

\footnotetext{
${ }^{\dagger}$ corresponding author, Tel:(212)327-8860, Fax:(212)327-8507, e-mail: slava@calif.rockefeller.edu
} 
methods. On the contrary, in the microcanonical ensemble, by tuning the energy, a system can be "forced" into these canonically unstable two-phase coexistence states. Reasons for this are simple: coexisting phases are separated by a boundary which carries a certain entropic cost which is determined by the depth of the dip $\delta s$. If a system is small or the interaction is long-range so that the interfacial region occupies a significant part of the total volume, this cost becomes important. Therefore if we put two weakly interacting small clusters that are allowed to exchange energy with each other into a state near the "bottom" of the dip, one of them will gain some energy and move close to the single-phase state 2 , and and the other will lose some energy and move towards another single-phase state 1 . As a result, the total entropy of two clusters will increase by $\approx 2 \delta s$. If a system consisting of many such energy-exchanging clusters is put into a state where each cluster initially has energy between $e_{1}$ and $e_{2}$, most probably it will arrange itself so that some cluster will be in state $e_{1}$ and the remaining ones in $e_{2}$. This scenario was frequently observed in Monte-Carlo simulations of small Lennard-Jones clusters [9, 10], where the probability to find a cluster with an interface was very low. On the contrary, in the ME one can tune the energy of a single cluster to any state including those in the phase coexistence interval and therefore put a system into a state with an interface even if it is entropically unfavorable.

Usually, as the system size increases and the contribution of the interface to the total entropy becomes negligible, the convex dip gets shallower and in the limit of infinite system size becomes a straight line. At this point phase coexistence states can be accessed by both ME and CE methods and two ensembles become equivalent. However, if the interaction potential of the system is sufficiently long-range (non-integrable at the large- $r$ limit), the relative interface contribution does not disappear in the bulk limit. In this case a system with a first-order phase transition in the ME can exhibit an entirely different phenomenology than in the CE. For example, microcanonical gravitating systems may have regions with negative specific heat even in the infinite system size limit [11,6). Similar to the small cluster case, these states with negative specific heat can not be observed in the equilibrium CE since they are unstable.

To illustrate both the short- and the long-range cases, we study the ferromagnetic $q$-state Potts model, defined by Hamiltonian (1).

$$
H=-\sum_{i<j} \delta_{s_{i}, s_{j}}, s_{k}=1, \ldots, q
$$

Two opposite limits are considered: Nearest Neighbor (NN) interaction as an example of a system with short-range interactions and the Mean Field (MF) theory as an example of a system with infinitely long-range interactions. In the first case, the sum in (11) runs only over nearest neighbor spins, in the second case, it includes all spins present in the system. In the MF model it is customary to introduce a $1 / N$ prefactor to make the total energy scale as $\sim N$ in the $N \rightarrow \infty$ limit. With $q>4$ for NN in 2 dimensions and $q>2$ for MF, both of these models have first-order phase transitions [12]. The $q=5 \mathrm{NN}$ model is studied via an exact microcanonical transfer matrix enumeration on a two-dimensional square lattice of up to $6 \times 6$ sites. The $q \geq 3 \mathrm{MF}$ models are studied both by exact enumeration and continuous integration for a finite number of spins; an analytical asymptotics for $s(e)$ is found in the infinite size limit.

Given all the benefits of ME approach to first-order phase transitions outlined above, a natural question, "what can be learned about the ME properties from known CE behavior" arises. To address this question, we consider a typical example of a system with a convex entropy dip and perform a transition from ME to $\mathrm{CE}$ and back. It has been already stated [2] that usually the ME data allow a rather precise determination of all the CE properties but not the inverse. The reason for this is that the inverse Laplace transform, which is performed to obtain the ME entropy from the canonical free energy, is much less numerically stable than the direct one. Moreover, in the last section of this paper we show that it is impossible to recover any information about the ME entropy convex dip from the corresponding CE free energy if it is known only in the $N \rightarrow \infty$ limit.

\section{EXACT ENUMERATION OF STATES FOR 2D POTTS MODEL}

Let us consider a 2D Potts model on a square lattice of the size $L \times L$. Since we are looking for signs of a first-order phase transition, we need to include into consideration at least the smallest possible value of $q$ for which such a transition exists in the bulk limit: $q=5$ [12]. A microcanonical transfer matrix method developed by Binder [13] is used. Cylindrical boundary conditions with open bonds at the top and the bottom are utilized, a choice that will be justified below. We first enumerate all possible states $|i\rangle, i=1, \ldots, q^{L}$ of a single 1-dimensional ring of spins, calculate their energies $E_{i}$, and define a transfer matrix $\hat{T}$ with the elements $T_{i j}$ equal to the energy of all bonds between two adjacent $1 \mathrm{D}$ rings of spins that are in $|i\rangle$ and $|j\rangle$ states. Then we define the degeneracy $D_{i}^{1}(E)$ of a state $|i\rangle$ with the energy $E_{i}$ of a $1 \mathrm{D}$ spin ring, $D_{i}^{1}(E)=\delta_{E, E_{i}}$. The degeneracy of state with the energy $E$ of a lattice consisting 
of a stack of $n$ rings where the state of the top ring is $i$ and the states of all lower rings are summed over, can be recursively expressed:

$$
D_{i}^{n}(E)=\sum_{j=1}^{q^{L}} D_{j}^{n-1}\left(E^{\prime}\right) \delta_{E, E^{\prime}+E_{j}+T_{j i}}
$$

We apply formula (2) $N-1$ times and finally obtain for the degeneracy of the whole lattice

$$
D(E)=\sum_{i=1}^{q^{L}} D_{i}^{n}(E) .
$$

This approach allows us to reduce the number of operations to $\sim L^{2} q^{2 L}$ compared to $\sim q^{L^{2}}$ as for a straightforward exact enumeration. However it requires enough memory to store the transfer matrix which has $q^{2 L}$ elements. Applying a similar method to a system on a torus (with completely periodic boundary conditions) will require storing a degeneracy matrix $D_{i j}^{n}(E)$ with $2 L^{2} q^{2 L}$ elements and $\sim L^{2} q^{3} L$ operations. Hence we chose to limit our considerations to systems with cylindrical boundary conditions, for which our computer resources allow us to study $q=5$ spin systems on lattices with the base of the cylinder (length of the ring) up to 5 . The main quantity we are looking at is the specific microcanonical entropy defined as the logarithm of the density of states $\Omega(E)$ (which coincides with the degeneracy $D(E)$ if the distance between energy levels is 1 ) divided by the number of spins $N=L \times L$ : $s(E) \equiv \ln (\Omega(E)) / N$, with $E$ being the total energy of the system For convenience we also use the intensive energy variable, energy per spin $e=E / N$. For the Hamiltonian (1) on the infinite 2D square lattice $e$ varies between -2 and 0 . Plots of specific entropy $s(e)$ as a function of $e$ for various lattice sizes for $q=5$ are presented in Figure 2 ; to be able to distinguish curves for different system sizes, we shifted plots for larger $\mathrm{N}$ upward.

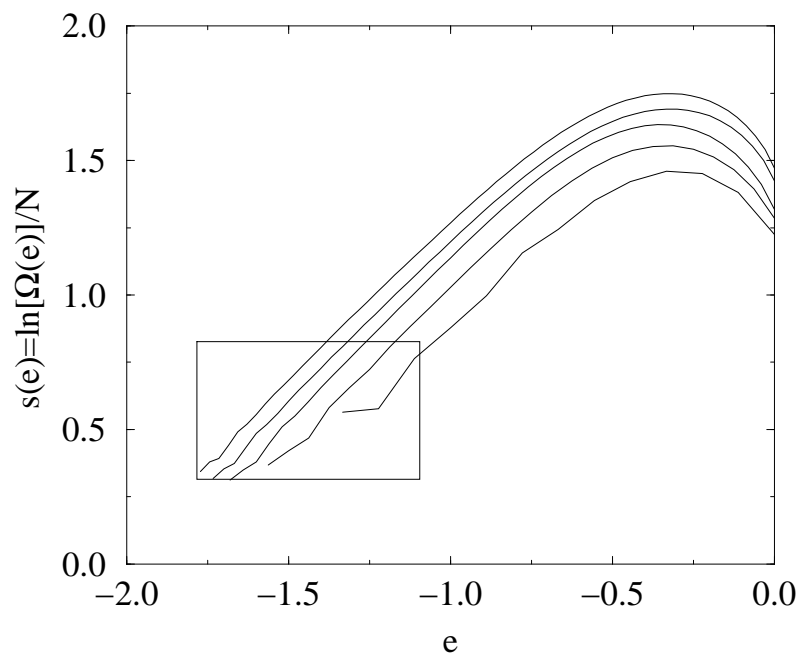

Fig. 2. Specific microcanonical entropy $s(e) \equiv \ln (\Omega(e)) / N$ vs. energy per spin $e$ for $q=5$ for (top to the bottom) $7 \times 5$, $6 \times 5$, $5 \times 5,4 \times 4$, and $3 \times 3$ lattices. A box is drawn around convex dips in all 5 curves. Ground states, which are separated from the first excited states by $\Delta E=3$ energy gap, are not shown in all curves.

Systems of all sizes (even with $N=9$ spins) exhibit definite signs of a phase transition: a dip between $e \approx-1.7$ and $e \approx-1.2$ can be clearly seen inside the box in all 5 plots. The dip gets shallower as $N$ increases, which is consistent with reduction of the relative entropic cost of the interface for bigger systems.

\section{MF POTTS MODELS}

Let us consider next a Mean Field Potts (MFP) model defined by the Hamiltonian

$$
H=\frac{-2}{N-1} \sum_{i=1}^{q} N_{i}\left(N_{i}-1\right) .
$$


Here $N_{i}$ is a number of spins in the ith state, $0 \leq N_{i} \leq N, 1 \leq i \leq q$. The coefficient $-2 /(N-1)$, specific for a 2D square lattice, is introduced to make the MF Hamiltonian consistent with the exact one (1): with this coefficient the energy per spin $e$ in a perfectly ordered configuration is also equal to -2 . One can view a MF model not only as an approximation to a real system, but also as an independent model with non-decaying infinitely-long interactions. It is known [12] that in the canonical ensemble the MF has a first-order phase transition for $q \geq 3$ and a second-order for $q=2$ (Mean Field Ising model).

The microcanonical partition function of the MF Potts model can be expressed as

$$
W(\tilde{E})=\sum_{N_{1}=0}^{N} \ldots \sum_{N_{q}=0}^{N} \frac{N !}{\prod_{i=1}^{q} N_{i} !} \delta_{\sum_{i=1}^{q} N_{i}, N} \delta_{\sum_{i=1}^{q} N_{i}^{2}, \tilde{E}}
$$

Here $\tilde{E} \equiv-E(N-1) / 2+N$ (c.f. (禹) is a rescaled energy of the system. The two Kronecker $\delta$ symbols are coming from the requirements that the total number of particles and the total energy are equal to $N$ and $\tilde{E}$, respectively; the factorial ratio gives the statistical weight of the state. To calculate the partition function, for moderate $N(N \leq 150)$ we perform an exact enumeration of states using integer arithmetics, while for larger $N(150<N \leq 600)$ we use rational number calculations. The results for the specific entropy $s(e)$ are presented in Figure 3.

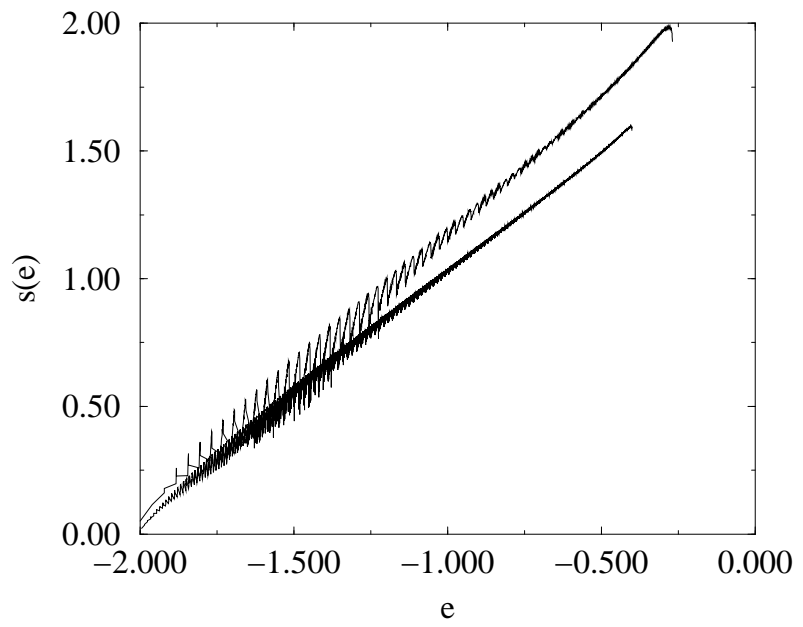

Fig. 3. Entropy $s(e)$ vs. energy per spin $e$ for $q=5$ and $N=500$ (line) and $q=7$ and $N=100$ (lower line).

Since energy states with high and low degeneracies are often observed to be close to each other, these plots have a rather rugged structure. It naturally leads to the assumption that only high-degeneracy states contribute noticeably to a smooth coarsegrained entropy, which is defined as the logarithm of the sum of the statistical weights of a group of states with energies falling into a bin $e-\Delta e / 2, e+\Delta e / 2$. The size of the bin $\Delta e$ must be large enough to include several energy states. Below we calculate the coarsegrained entropy and the leading term of its asymptotic behavior for $N \rightarrow \infty$.

After replacing sums by integrals, factorials by continuous functions via Stirling's formula, and introducing the intensive variables $n_{i} \equiv N_{i} / N, \tilde{e} \equiv \tilde{E} / N^{2}$, Eq. (5) becomes:

$$
W(\tilde{e}) \approx N^{q-3} \int_{0}^{1} \ldots \int_{0}^{1} \delta\left(\sum_{i=1}^{q} n_{i}-1\right) \delta\left(\sum_{i=1}^{q} n_{i}^{2}-\tilde{e}\right) \exp \left(-N \sum_{i=1}^{q} n_{i} \ln n_{i}\right) d n_{1} \ldots d n_{q}
$$

Two integrations which eliminate the $\delta$-functions are easily performed by introducing hyperspherical coordinates with the radius $r=\sqrt{\sum_{i=1}^{q} n_{i}^{2}}$ and the polar axis $x_{1}=r \cos \left(\phi_{1}\right)$ along the $q$-dimensional vector $(1 / \sqrt{q}, 1 / \sqrt{q}, \ldots, 1 / \sqrt{q})$. The Jacobian of this transformation is given by $r^{q-1} \prod_{i=1}^{q-1}\left[\sin \left(\phi_{i}\right)\right]^{q-i-1}$ (see, for example, [14]).

$$
W(\tilde{e}) \approx \theta(\tilde{e}-1 / q) \frac{\left[N^{2}(\tilde{e}-1 / q)\right]^{\frac{q-3}{2}}}{2 \sqrt{q}} \int \exp \left(-N \sum_{i=1}^{q} n_{i} \ln n_{i}\right) \prod_{i=1}^{q} \theta\left(n_{i}\right) d^{q-2} \Omega,
$$

where $\theta(x)$ is the unit step function, and $d^{q-2} \Omega$ is the remaining part of hyperspherical $q-1$ dimensional angular differential after the polar angle $\phi_{1}$ integration has been carried out. The integration in (7) runs over the remaining 
angular variables in the first quadrant (only where all $n_{i}$ are non-negative) and is performed numerically. For the simplest nontrivial case of $q=3$, the above expression transforms to the following one-dimensional integral: (see Fig. 4)

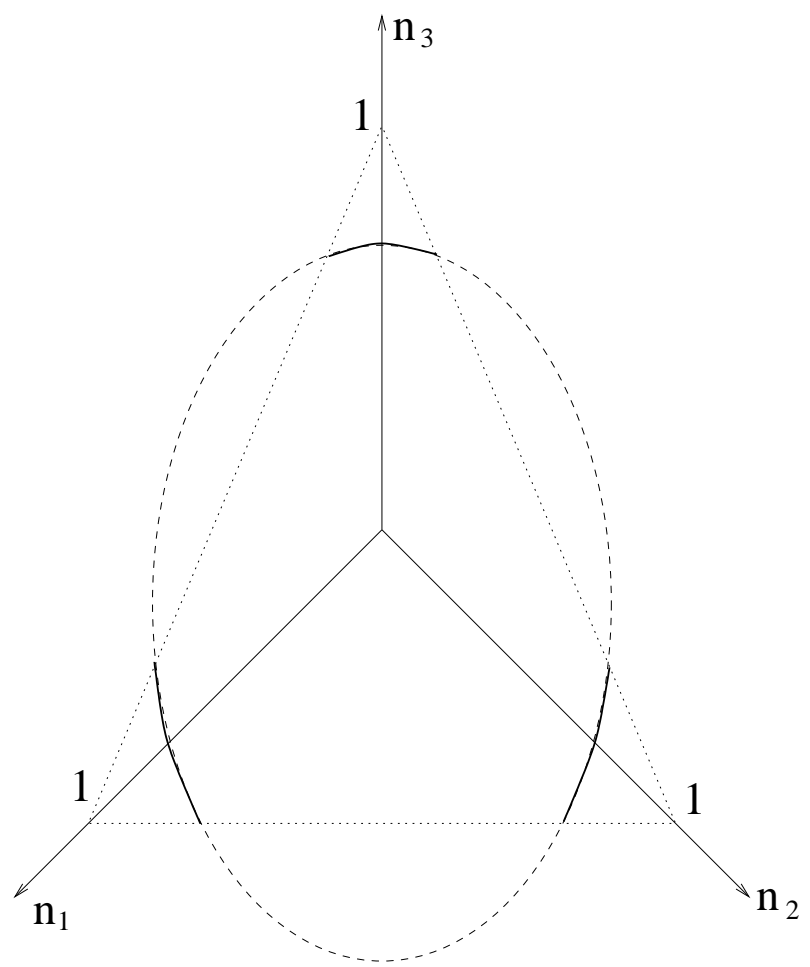

Fig. 4. Sketch of integration contour for (8) for $q=3 \mathrm{MF}$ Potts model (3 solid lines segments). They lie on the intersection of a circle with center $\left(\frac{1}{\sqrt{3}}, \frac{1}{\sqrt{3}}, \frac{1}{\sqrt{3}}\right)$ and radius $r=\sqrt{\tilde{e}-1 / 3}$ with the plane $n_{1}+n_{2}+n_{3}=1$.

$$
W(\tilde{e}) \approx \theta(\tilde{e}-1 / 3) \frac{1}{2 \sqrt{3}} \int_{0}^{2 \pi} \exp \left(-N \sum_{i=1}^{q} n_{i} \ln n_{i}\right) \prod_{i=1}^{3} \theta\left(n_{i}\right) d \phi,
$$

where

$$
\begin{gathered}
n_{1}=\frac{1}{3}-\rho\left(\frac{\cos \phi}{\sqrt{6}}+\frac{\sin \phi}{\sqrt{2}}\right) \\
n_{2}=\frac{1}{3}-\rho\left(\frac{\cos \phi}{\sqrt{6}}-\frac{\sin \phi}{\sqrt{2}}\right) \\
n_{3}=\frac{1}{3}+2 \rho \frac{\cos \phi}{\sqrt{6}} \\
\rho=\sqrt{\tilde{e}-1 / 3} .
\end{gathered}
$$

In the limit of $N \rightarrow \infty$ the integral (7) can be evaluated asymptotically for any $q$. With the restrictions $\sum_{i=1}^{q} n_{i}=1$ and $\sum_{i=1}^{q} n_{i}^{2}=\tilde{e}$, the function $\sum_{i=1}^{q} n_{i} \ln n_{i}$ has $q$ identical maxima at which one $n_{i}$ (say, $n_{1}$ ) is large, and all the other $n_{i}$ are the same:

$$
n_{1}^{*}=\frac{1+\sqrt{(q \tilde{e}-1)(q-1)}}{q}, n_{2}^{*}=\ldots=n_{q}^{*}=\frac{1-\sqrt{(q \tilde{e}-1) /(q-1)}}{q}
$$

To illustrate the dominance of the contributions from the maxima (10), we present in Fig. 5 a magnified segment of the plot of a non-coarsegrained (exact) $s(\tilde{e})$ for $q=5$ and $N=500$ from Fig. 3, together with a sequence of points $\left(e_{K}, s_{K}\right)$ that are the maxima defined by (10)for integer $N_{i}$ :

$$
e_{K}=\frac{-2}{N(N-1)}\left[K^{2}+\frac{(N-K)^{2}}{q-1}-N\right], s_{K}=-\frac{K}{N} \ln \frac{K}{N}-\frac{N-K}{N} \ln \frac{N-K}{N(q-1)},
$$

where $K=N / q, N / q+1, \ldots, N$ labels the maxima. 


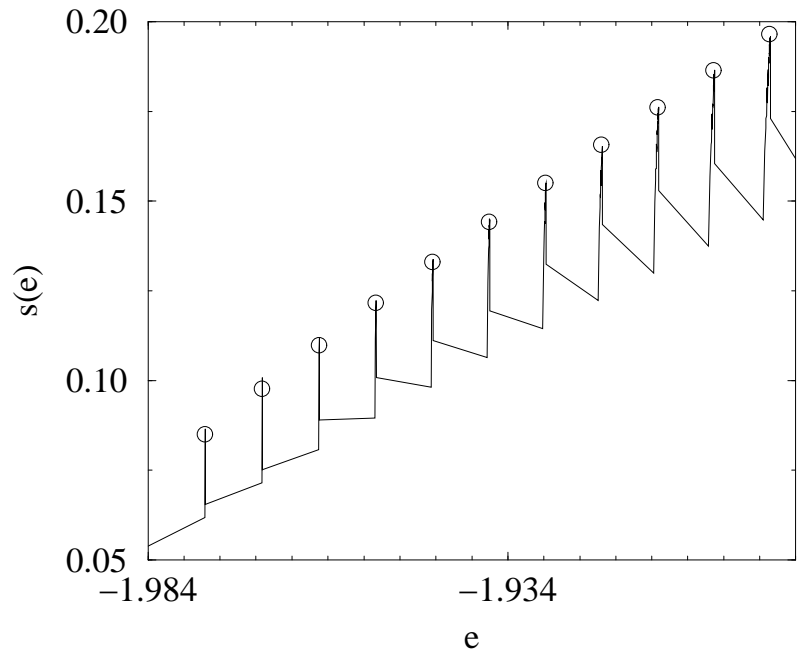

Fig. 5. Entropy $s(e)$ vs. energy per spin $e$ for $q=5$ and $N=500$ (solid line) and contribution from the states defined by Eq. (11) (circles).

Evaluating the integrals in (6) by the steepest descent method around the maxima (10), we obtain for the specific entropy:

$$
s(\tilde{e})=\ln (W(\tilde{e})) / N=-\sum_{i=1}^{q} n_{i}^{*} \ln n_{i}^{*}+\mathcal{O}\left(\frac{\ln (N)}{N}\right) .
$$

Now we proceed by looking at signs of the convex dip in $s(e)$ and associated with it an interval with positive second derivative $s^{\prime \prime}(e)$, where the microcanonical specific heat $C_{v}=-\left(s^{\prime}(e)\right)^{2} / s^{\prime \prime}(e)$ is negative. Generally, a deviation of the $s(e)$ plot from a straight line is hardly visible, so we present the results through the derivative $s^{\prime}(e)$. This is done for a twofold purpose: first, a sign of the second derivative can be easily deduced from the plots of $s^{\prime}(e)$; second, recalling the definition of the microcanonical inverse temperature $\beta(e) \equiv s^{\prime}(e)$, the $s^{\prime}(e)$ is of interest by itself. In Figure 6 we present the plot of $s^{\prime}(e)$ for the $q=3 \mathrm{MFP}$ model for various numbers of spins $N$ ranging from 50 to infinity. Curves for finite $N$ are produced by direct numerical evaluation of the integral (6); knowledge of the $N \rightarrow \infty$ asymptotics (12) helps to avoid real number overflow for large $N$. The bottom line for $N=\infty$ is the exact steepest descent result (12).

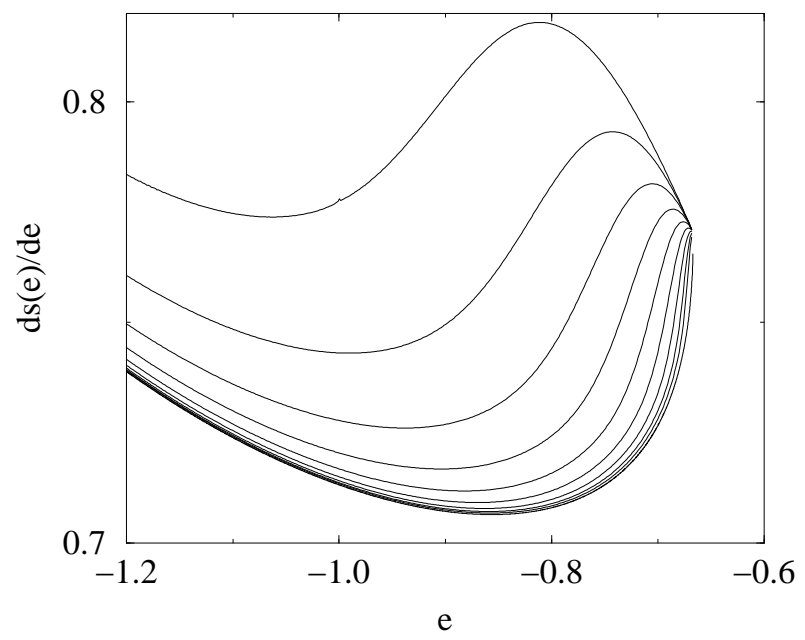

Fig. 6. Specific entropy derivative $s^{\prime}(e)$ vs. energy per spin $e$ for $q=3$ and for 10 various $N$ (going from the up-most line down) $N=50,100,200,400,800,1600,3200,6400,12800, \infty$.

For all system sizes $N, s^{\prime}(e)$ approaches the same value in the high-energy limit, $s^{\prime}(e=-2 / 3)=3 / 4$ (c.f. (8), (12). For any finite $N$, the second derivative of the entropy also approaches a finite negative value, $s^{\prime \prime}(e) \rightarrow-9 / 16$, 
as $e \rightarrow-2 / 3$, but the interval near $e=-2 / 3$, where $s^{\prime \prime}(e)$ is negative, shrinks as $1 / \sqrt{N}$.

In Figure $7, s^{\prime}(e)$ is plotted for various $q$ ranging between 2 and 100 for an infinite system.

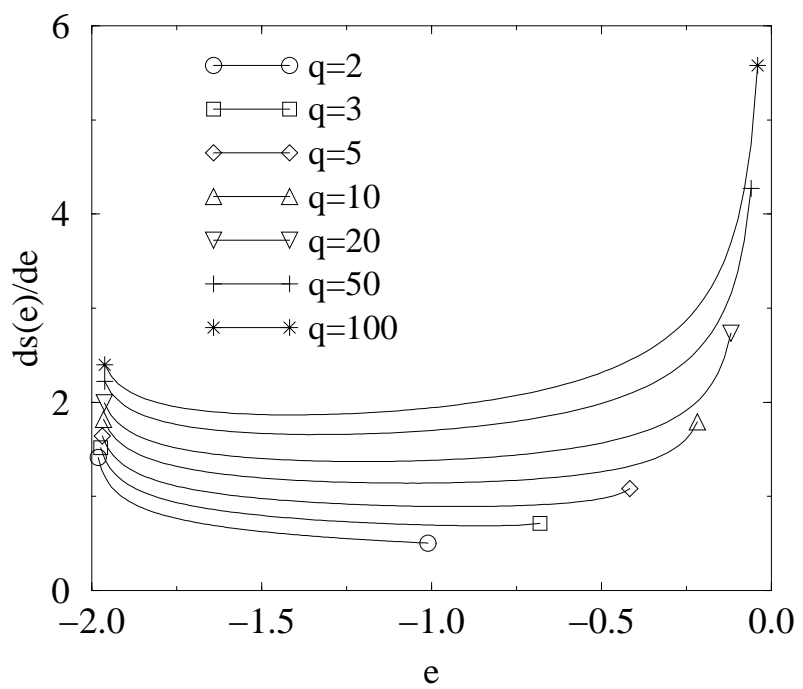

Fig. 7. Entropy derivative $s^{\prime}(e)$ vs. energy per spin $e$ for $N=\infty$ and for 7 values of $q$ (going from the upper line down) $q=100,50,20,10,5,3,2$.

For $q=2$, which corresponds to the Ising mean-field model, $s^{\prime \prime}(e)$ is negative for all allowed energies. This is consistent with the canonical ensemble results for the MF Potts $q=2$ model, where the specific heat has finite limits on both sides of a second-order phase transition and experiences a discontinuous jump at the phase transition point. The microcanonical inverse temperature $\beta=s^{\prime}(e)$ at the point of phase transition $e=-1$, which is the high end point of the allowed energy interval $-2 \leq e \leq 2 / q$, is equal to its MF Potts canonical equivalent, $\beta_{c}=1 / 2$, given that the energy scale is defined as in the Hamiltonian (11). For $q \geq 3$, a region of positive $s^{\prime \prime}(e)$ appears and gets wider with increasing $q$. At the upper boundary of the allowed energy interval, $e=-2 / q$, the entropy derivative grows linearly with $q, s^{\prime}(e=-2 / q)=q / 4$.

Figures 6 and 7 indicate that MF Potts model does exhibit a convex dip in the entropy plot and an associated interval with negative specific heat, even in the infinite system limit. The reason for this is the distance-independent nature of interactions in the MF Hamiltonian which makes phase segregation impossible. Hence the entropic cost of phase coexistence scales linearly with the number of spins in the system and the depth of the convex dip in the entropy plot goes to a finite limit as $N \rightarrow \infty$.

\section{CONNECTION TO CANONICAL ENSEMBLE}

In the previous sections we performed a microcanonical analysis of the Potts model which is one of the most simple systems with a first-order phase transition. However, in general the canonical properties are much better studied than the microcanonical ones. A question then arises: what can be learned about the microcanonical behavior of a system which has a first-order phase transition from its canonical solution? A general answer is well-known: if a system is infinite and extensive, i.e. the interaction is not long-range, then microcanonical and canonical behavior are the same. However, the MF Potts model can be considered as having infinitely long-range interactions and, as we showed above, does have a negative specific heat even in the infinite system size limit. Below we present a description of what happens to the negative specific heat interval and the convex dip in the entropy plot as a whole, if we formally attempt a transformation from the microcanonical ensemble to the canonical ensemble (i.e. a Laplace transform of microcanonical partition function) and then transform back from the canonical to the microcanonical ensemble (i.e. inverse Laplace transform of the canonical partition function). This procedure discussed below is of a rather general nature and can be applied to any system with a convex dip in the microcanonical entropy plot; to the best of our knowledge, such a general description has yet not been presented in the literature.

To calculate the canonical partition function $Z(\beta)$,

$$
Z(\beta)=N \int e^{N[s(e)-\beta e]} d e,
$$


let us consider an interval of energies where the specific entropy $s(e)$ has a convex dip (Fig. 8):

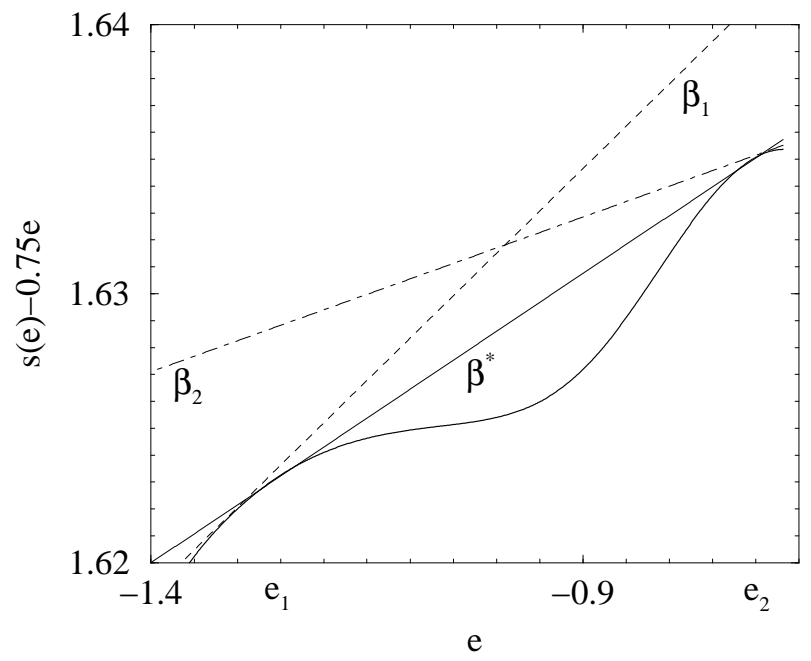

Fig. 8. Rescaled MFP entropy $s(e)-0.75 e$ vs. energy $e$ for $q=3$ and $N=50$ (black solid line). Straight lines $\beta e+\alpha$ have slopes: $\beta_{1}=0.7815, \beta_{2}=0.7615$, and $\beta^{*}=0.7715$.

For $N \rightarrow \infty$, the integral (13) can be evaluated by the saddle point method which yields for the specific canonical free energy $f(\beta)$

$$
f(\beta) \equiv-\frac{1}{\beta N} \ln [Z(\beta)]=e^{*}-\frac{s\left(e^{*}\right)}{\beta}+\mathcal{O}\left(\frac{\ln N}{N}\right)
$$

where $e^{*}=e^{*}(\beta)$ is the solution of equation

$$
s^{\prime}\left(e^{*}\right)=\beta,
$$

that maximizes the expression in the exponential of (13). For a concave entropy $s(e)$, Eq. (15) has not more than one solution. For a system where the entropy has a convex dip, for a certain $\beta$, Eq. (15) may have two solutions, $e_{1}$ and $e_{2}$. This is the case for all three $\beta$ shown in Fig. 8. However, for both $\beta_{1}$ and $\beta_{2}$ a contribution from the second, smaller, maximum of $s(e)-\beta e$ to the integral (13) is exponentially small in $N$. For example, for $\beta_{1}$ the left maximum $e_{1}$ is the global one, and

$$
f\left(\beta_{1}\right) \approx e_{1}-\frac{s\left(e_{1}\right)}{\beta_{1}}-\frac{e^{N\left[\left(s\left(e_{2}\right)-\beta_{1} e_{2}\right)-\left(s\left(e_{1}\right)-\beta_{1} e_{1}\right)\right]}}{N} .
$$

Only for $\beta=\beta^{*}$, do both maxima give equal contributions to $f\left(\beta^{*}\right)$; $\beta^{*}$ can then be identified with the inverse temperature of the first-order phase transition in the canonical ensemble. It follows from (14, 15) that

$$
\left.\frac{d[\beta f(\beta)]}{d \beta}\right|_{\beta \neq \beta^{*}}=e^{*},\left.\frac{d[\beta f(\beta)]}{d \beta}\right|_{\beta=\beta^{*}+0}=e_{1},\left.\frac{d[\beta f(\beta)]}{d \beta}\right|_{\beta=\beta^{*}-0}=e_{2},
$$

which is in agreement with Ehrenfest's definition of a first-order phase transition.

To put it another way, by performing the integration (14) for various $\beta$, we sample $s(e)$ for all $e$ except for those inside a convex dip, and this sampling becomes a local one-to-one $e \rightarrow \beta$ correspondence for infinite $N$. On the contrary, the contribution of a convex dip to $f(\beta)$ is exponentially (in $N$ ) suppressed and vanishes completely for $N \rightarrow \infty$.

Now we have arrived to where we usually start from: a canonical ensemble specific free energy $f(\beta)$ calculated in the $N \rightarrow \infty$ limit. It is a continuous function of $\beta$ with a jump discontinuity in its first derivative at the first-order transition point $\beta^{*}$. As we showed above, it contains no information about the behavior of $s(e)$ in the interval where $s(e)$ is convex. However, it is still worth finding out what kind of microcanonical entropy can be reconstructed from $f(\beta)$. In order to do that we perform an inverse Laplace transformation of $f(\beta)$ :

$$
\tilde{s}(e)=\frac{1}{N} \ln \left\{\frac{1}{2 \pi i} \int_{\epsilon-i \infty}^{\epsilon+i \infty} e^{N \beta[e-f(\beta)]} d \beta\right\}
$$


In the limit of $N \rightarrow \infty$, we look for stationary points of the expression in the exponential, $\beta e-\beta f(\beta)$. Since for $\beta \neq \beta^{*}, d[\beta f(\beta)] / d \beta=e^{*}(\beta)$, the extremum of the exponential is reached when $e^{*}(\beta)=e$. Taking into account (14, 15), we obtain that $\tilde{s}(e)=s(e)$. This is true for $e \leq e_{1}$ and $e \geq e_{2}$. Since $f(\beta)$ has a cusp at $\beta=\beta^{*}$ and its leftand right- hand side derivatives are given by (17), the derivatives of $\beta[e-f(\beta)]$ at $\beta=\beta^{*}$ for $e_{1}<e<e_{2}$ are:

$$
\left.\frac{d[\beta e-\beta f(\beta)]}{d \beta}\right|_{\beta=\beta^{*}-0}=e-e_{2}<0,\left.\frac{d[\beta e-\beta f(\beta)]}{d \beta}\right|_{\beta=\beta^{*}+0}=e-e_{1}>0 .
$$

Then for $e_{1}<e<e_{2}, \beta^{*}$ gives the minimum of $\beta[e-f(\beta)]$ and in the saddle point approximation, integral (18) is reduced to

$$
\tilde{s}(e)=\beta^{*}\left[e-f\left(\beta^{*}\right)\right]+\mathcal{O}\left(\frac{\ln (N)}{N}\right)
$$

From the definition of $\beta^{*}$ (see Fig. 8) we have that $\beta^{*}\left(e_{1}-e_{2}\right)=s\left(e_{1}\right)-s\left(e_{2}\right)$ and $\beta^{*} f\left(\beta^{*}\right)=\beta^{*} e_{1}-s\left(e_{1}\right)$. Substituting this into 20], one obtains for $e_{1}<e<e_{2}$ :

$$
\tilde{s}(e)=\frac{e-e_{1}}{e_{2}-e_{1}}\left[s\left(e_{2}\right)-s\left(e_{1}\right)\right]+s\left(e_{1}\right)+\mathcal{O}\left(\frac{\ln (N)}{N}\right) .
$$

So in a double transformed microcanonical entropy a convex dip is replaced by a straight line (marked $\beta^{*}$ in Fig. 8).

For any sufficiently smooth and not too rapidly growing function, application of the direct and inverse Laplace transforms should leave this function unchanged. However, exact canonical solutions are almost never available for finite $N$; more often they are known in the infinite system limit which is singular. As was shown above, if the $N \rightarrow \infty$ limit is taken before the integral (18) is evaluated, all information about a possible convex intruder in $s(e)$ is totally lost. Assume we study $f(\beta)$ numerically for finite $N$. Still, our chances to recover information about a convex dip in $s(e)$ via the inverse Laplace transform of the CE partition function are quite low even for modest $N$ since the contribution of the convex dip to $f(\beta)$ is exponentially small in $N$. In statistical terms, it can be rephrased as exponentially small accessibility of low statistical weight states to canonical random sampling.

For extensive systems with first-order phase transitions, when the contribution of the interfacial states to $s(e)$ disappears as $N \rightarrow \infty$, a convex intruders flattens into a straight line. In this case a microcanonical behavior can be reconstructed from a known canonical solution via the procedure outlined above. In fact, this reconstruction can be made operating solely with intensive quantities $f(\beta)$ and $s(e)$ via what can be called a generalized Legendre transform:

$$
\tilde{s}(e)=\beta_{e} e-\beta_{e} f\left(\beta_{e}\right),\left.\frac{d[\beta f(\beta)]}{d \beta}\right|_{\beta=\beta_{e}}=e .
$$

The second equation can only be solved for $e<e_{1}$ and $e>e_{2}$. For $e_{1}<e<e_{2}$ these two branches of $s(e)$ are connected by a straight line (cf. (21)).

\section{CONCLUSION}

In two examples of the Potts model considered above, NN and MF, there is a convex dip in the entropy plot when the system size is finite. In the short-range-interacting NN model this dip flattens to a straight line as the system size tends to infinity, while the dip in the entropy of the infinitely-long-interacting MF model persists in this limit. This difference in behavior between $\mathrm{NN}$ and MF models is caused by different relative entropic cost of interfacial states which scales with the number of spins $N$ as $N^{\frac{d-1}{d}}$ in the locally interacting models such as NN and goes to a constant in the MF model. Since a convex dip in the microcanonical entropy is always associated with a negative specific heat interval, the corresponding states in the canonical ensemble are thermodynamically unstable and are not represented in the canonical free energy. Therefore in small or long-range interacting systems, these states of phase coexistence can only be studied in the framework of the microcanonical ensemble.

[1] R. W. Gerling and A. Hüller, Z. Phys. B 90, 207 (1993). 
[2] A. Hüller, Z. Phys. B 93, 401 (1994).

[3] D. J. Wales and R. S. Berry, Phys. Rev. Lett. 73, 2875 (1994).

[4] P. Labastie and R. L. Whetten, Phys. Rev. Lett. 65, 1567 (1990).

[5] M. Bixon and J. Jortner, J. Chem. Phys. 91, 1631 (1989).

[6] D. Lynden-Bell, Physica A 263, 293 (1999).

[7] D. H. E. Gross and E. V. Votyakov, Eur. Phys. J. B 15, 115 (2000).

[8] D. H. E. Gross, M. E. Madjet, Z. Physik B 104, 541 (1997).

[9] D. Wales and J. P. K. Doye, J. Chem. Phys. 103, 3061 (1995).

[10] R. M. Lynden-Bell, D. J. Wales, J. Chem. Phys. 101, 1460 (1994).

[11] T. Padmanabhan, Phys. Rep. 188, 285 (1990).

[12] F. Y. Wu, Rev. Mod. Phys. 54, 235 (1982).

[13] K. Binder, Physica, 62, 508 (1972).

[14] G. Baley Price, Multivariable Analysis, Springer-Verlag, New York, 1984. 